Bull. Chem. Soc. Ethiop. 2013, 27(1), 69-76.

Printed in Ethiopia

DOI: http://dx.doi.org/10.4314/bcse.v27i1.7

ISSN 1011-3924

(c) 2013 Chemical Society of Ethiopia

\title{
A NOVEL PROTON TRANSFER COMPOUND (A NEW MOLYBDATE SALT) AND ITS X-RAY STRUCTURE
}

\author{
Iran Sheikhshoaie ${ }^{*}$ and Mahdeieh Ghazizadeh \\ Department of Chemistry, Shahid Bahonar University of Kerman, Iran
}

(Received November 22, 2011; revised August 14, 2012)

\begin{abstract}
A novel proton transfer compound $\left(\mathrm{OHRNH}_{3}\right)_{2}{ }^{2+}\left(\mathrm{MoO}_{4}\right)^{2-}(\mathrm{R}=2$-methyl-1-propyl), derived from 2-amino-2-methyl-1-propanol and $\mathrm{MoO}_{2}(\text { acac })_{2}$, synthesized and characterized by ${ }^{1} \mathrm{H}$ NMR, X-ray diffraction analysis, UV-Vis and FT-IR spectroscopy. The single crystal X-ray diffraction analysis revealed that intra- and intermolecular proton transfer from $\left(\mathrm{MoO}_{4} \mathrm{H}_{2}\right)$ to $\left(\mathrm{OHRNH}_{2}\right)$ results in the formation of a new molybdate salt that its fragments are connected through $\mathrm{H}$-bonding and ion-pairing as shown in the X-ray crystal structure. This salt crystallizes in the space group $P 2{ }_{1} / n \mathrm{P}_{-} 1$ of the monoclinic system, with four molecules per unit cell. The unit cell parameters are $\mathrm{a}=13.6091(11) \AA, \mathrm{b}=6.1049(5) \AA, \mathrm{c}=17.0840(13) \AA$ and $\beta=97.745(4)^{\circ}$.
\end{abstract}

KEY WORDS: Proton transfer, Molybdate salt, X-ray structure, $\mathrm{MoO}_{2}(\mathrm{acac})_{2}$, 2-Amino-2-methyl-1-propanol

\section{INTRODUCTION}

The different aspects of proton transfer systems have been studied by chemists in the recent years [1-5]. An interesting report in this area was investigation of the mechanism of proton transfer from intra-molecularly hydrogen-bonded acids and differences between nitrogen-tooxygen and nitrogen-to-nitrogen proton transfer [6, 7]. Whenever the hydrogen bonding associations result in complete proton transfer to the nitrogen atom, an ionic self-assembled compound is produced [8] and considerable stability upon structure-making process which can be used in designing of novel layered crystalline material is achieved $[9,10]$. Here we report the synthesis, characterization, and X-ray crystal structures of a molybdenum compound as a new molybdate salt.

\section{EXPERIMENTAL}

Materials and instruments

All the chemicals were purchased from Merck Company, and were used as such. UU-Vis spectra were recorded on a Perkin Elmer Lambda25 in the range of 200-700 nm. FT-IR spectra obtained as potassium bromide pellets in the range of $400-4000 \mathrm{~cm}^{-1}$ with a Nicolet-Impact 400D spectrometer. NMR spectra were recorded on Bruker advance DPX $400 \mathrm{MHz}$ instrument.

Syntheses of the proton transfer compound

This compound was synthesized by refluxing the solutions of equimolar quantities of $\mathrm{MoO}_{2}(\mathrm{acac})_{2}$ with 2-amino-2-methyl-1-propanol in methanol as solvent. $1.0 \mathrm{mmol}(0.3270 \mathrm{~g})$ of $\mathrm{MoO}_{2}(\mathrm{acac})_{2}$ in methanol was added to the boiling methanolic solution of $1.0 \mathrm{mmol}(0.10 \mathrm{~mL}$, $0.0891 \mathrm{~g}$ ) of 2-amin0-2-methyl-1- propanol in a $25 \mathrm{~mL}$ two-necked flask and reflux for $4 \mathrm{~h}$ and then left to stand (Scheme 1). The suitable yellow crystals (block, colourless) for X-ray analysis were obtained after two weeks. M.p. $=234{ }^{\circ} \mathrm{C}$ (with decomposition).

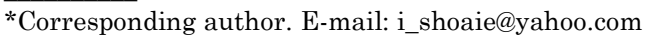




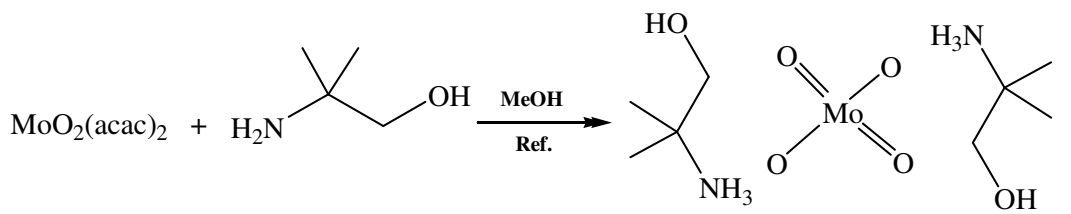

Scheme 1. The synthetic scheme for the preparation of $\left(\mathrm{OHRNH}_{3}\right)_{2}{ }^{2+}\left(\mathrm{MoO}_{4}\right)^{2-}(\mathrm{R}=2$-methyl-1propyl) compound.

$X$-ray structure analysis

Data collection: APEX2 (Bruker, 2009); cell refinement: APEX2 (Bruker, 2009); data reduction: APEX2 (Bruker, 2009); program(s) [11] used to solve structure: SHELXS97 (Sheldrick, 2008); program(s) used to refine structure: SHELXL97 (Sheldrick, 2008); molecular graphics: SHELXTL (Sheldrick, 2008) [12]. All esds (except the esd in the dihedral angle between two 1.s. planes) are estimated using the full covariance matrix. The cell esds are taken into account individually in the estimation of esds in distances, angles and torsion angles; correlations between esds in cell parameters are only used when they are defined by crystal symmetry. An approximate (isotropic) treatment of cell esds is used for estimating esds involving 1.s. planes. The weighted R-factor $\mathrm{wR}$ and goodness of fit $\mathrm{S}$ are based on $\mathrm{F}^{2}$, conventional $\mathrm{R}$-factors $\mathrm{R}$ are based on $F$, with $F$ set to zero for negative $F^{2}$. The threshold expression of $F 2>2$ sigma $\left(\mathrm{F}^{2}\right)$ is used only for calculating R-factors (gt) etc. and is not relevant to the choice of reflections for refinement. R-factors based on $\mathrm{F}^{2}$ are statistically about twice as large as those based on $\mathrm{F}$, and R-factors based on ALL data will be even larger. Selected crystals were mounted on a Bruker APEX-II CCD diffractometer with an APEX CCD area detector. A graphite-monochromatic Mo-K $\alpha$ radiation $(0.71073 \AA$ ) was used for all measurements. The nominal crystal-to-detector distance was $5.00 \mathrm{~cm}$. A hemisphere of data was collected by a combination of three sets of exposures at $T=93 \mathrm{~K}$. The structure was solved by direct methods (SHELXS97) [17]. Refinement was carried out with the full-matrix least-squares method based on F2 (SHELXL97) with anisotropic thermal parameters for all non-hydrogen atoms. The numbering scheme, ORTEP, of compound is shown in Figure 1, and its packing is shown in Figure 2. A summary of crystallographic data and selected bond lengths and angles are reported in Tables 1, respectively. Hydrogen atoms were inserted in calculated positions and refined riding with the corresponding atom. Further crystallographic data of the measurements are listed in Table 1. Some selected bond lengths $(\AA)$ and angles $\left({ }^{\circ}\right)$ for this structure were shown in Table 2. Some selected bond angles $(\AA)$ and bond lengths $\left(^{\circ}\right)$ for this compound are shown in Table 3. 


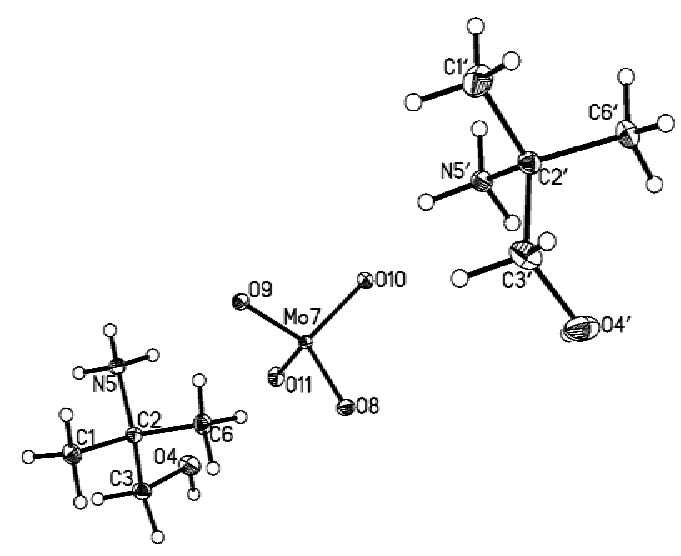

Figure 1. Molecular structure and atomic numbering for $\left(\mathrm{OHRNH}_{3}\right)_{2}{ }^{2+}\left(\mathrm{MoO}_{4}\right)^{2-}(\mathrm{R}=2$-methyl-1propyl) as a new molybdate salt (displacement ellipsoids are drawn at the $50 \%$ probability level).

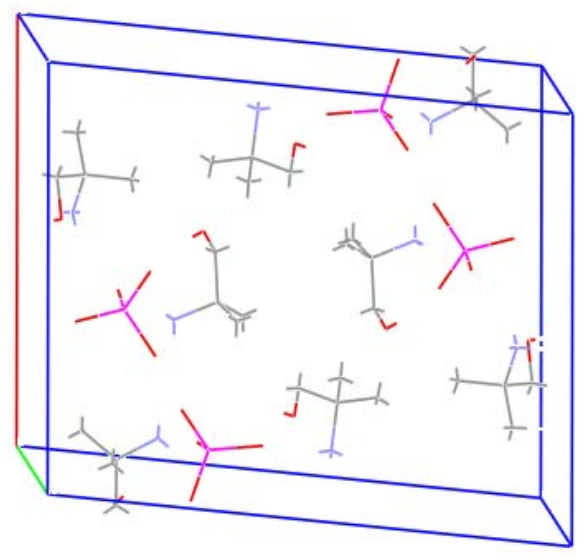

Figure 2. The crystal packing viewed along the $\mathrm{b}$ axis for $\left(\mathrm{OHRNH}_{3}\right)_{2}{ }^{2+}\left(\mathrm{MoO}_{4}\right)^{2-}(\mathrm{R}=2$-methyl1 -propyl) as a new molybdate salt.

Table 1. Crystallographic data of $\left(\mathrm{OHRNH}_{3}\right)_{2}{ }^{2+}\left(\mathrm{MoO}_{4}\right)^{2-}(\mathrm{R}=2$-methyl-1-propyl $)$ as a new molybdate salt.

\begin{tabular}{|l|l|}
\hline $2\left(\mathrm{C}_{4} \mathrm{H}_{12} \mathrm{NO}\right) \cdot \mathrm{MoO}_{4}$ & $F(000)=704$ \\
\hline$M_{r}=340.23$ & $D_{\mathrm{x}}=1.607 \mathrm{Mg} \mathrm{m}^{-3}$ \\
\hline Monoclinic, $P 2_{1} / n$ & Mo $K_{\alpha}$ radiation, $\lambda=0.71073 \AA$ \\
\hline Hall symbol: $-\mathrm{P} 2 \mathrm{yn}$ & Cell parameters from 9824 reflections \\
\hline$a=13.6091(11) \AA$ & $\theta=2.4-36.2^{\circ}$ \\
\hline$b=6.1049(5) \AA$ & $\mu=0.95 \mathrm{~mm}^{-1}$ \\
\hline$c=17.0840(13) \AA$ & $T=93 \mathrm{~K}$ \\
\hline$\beta=97.745(4)^{\circ}$ & Block, colourless \\
\hline$V=1406.43(19) \AA^{3}$ & $0.58 \times 0.24 \times 0.14 \mathrm{~mm}$ \\
\hline$Z$ & 4 \\
\hline
\end{tabular}

Bull. Chem. Soc. Ethiop. 2013, 27(1) 


\begin{tabular}{|l|l|}
\hline \multicolumn{2}{|c|}{ Data collection } \\
\hline Bruker APEX-II CCD diffractometer & 6756 independent reflections \\
\hline Radiation source: fine-focus sealed tube & 6034 reflections with $I>2 \sigma(I)$ \\
\hline graphite & $R_{\text {int }}=0.030$ \\
\hline$\varphi$ and $\omega$ scans & $\theta_{\max }=36.4^{\circ}, \theta_{\min }=2.4^{\circ}$ \\
\hline $\begin{array}{l}\text { Absorption correction: multi-scan } \\
S A D A B S \text { (Bruker, 2009) }\end{array}$ & $h=-22 \rightarrow 22$ \\
\hline$T_{\min }=0.609, T_{\max }=0.879$ & $k=-10 \rightarrow 10$ \\
\hline 45132 measured reflections & $l=-28 \rightarrow 28$ \\
\hline & Refinement \\
\hline Refinement on $F^{2}$ & $\begin{array}{l}\text { Primary atom site location: structure-invariant direct } \\
\text { methods }\end{array}$ \\
\hline$R\left[F^{2}>2 \sigma\left(F^{2}\right)\right]=0.021$ & $\begin{array}{l}\text { Hydrogen site location: inferred from } \\
\text { neighbouring sites }\end{array}$ \\
\hline$w R\left(F^{2}\right)=0.054$ & $\mathrm{H}$-atom parameters constrained \\
\hline$S=1.06$ & $\begin{array}{l}w=1 /\left[\sigma^{2}\left(F_{\mathrm{o}}^{2}\right)+(0.0239 P)^{2}+0.5911 P\right] \\
\text { where } P=\left(F_{\mathrm{o}}^{2}+2 F_{\mathrm{c}}{ }^{2}\right) / 3\end{array}$ \\
\hline 6756 reflections & $(\Delta / \sigma)_{\max }=0.002$ \\
\hline 160 parameters & $\Delta>_{\max }=0.65$ e $\AA^{-3}$ \\
\hline 0 restraints & $\Delta>_{\min }=-0.93$ e $\AA^{-3}$ \\
\hline &
\end{tabular}

Table 2. Selected bond angles $(\AA)$ and bond angles $\left({ }^{\circ}\right)$ for $\left(\mathrm{OHRNH}_{3}\right)_{2}{ }^{2+}\left(\mathrm{MoO}_{4}\right)^{2-}(\mathrm{R}=2$-methyl-1-propyl $)$ as a new molybdate salt.

\begin{tabular}{|c|c|c|c|}
\hline \multicolumn{4}{|c|}{ Bond length $(\AA)$} \\
\hline $\mathrm{C} 1-\mathrm{C} 2$ & $1.5253(15)$ & Mo7-O8 & $1.7695(8)$ \\
\hline $\mathrm{C} 1-\mathrm{H} 1 \mathrm{~A}$ & 0.9800 & Mo7-O10 & $1.7731(8)$ \\
\hline $\mathrm{C} 1-\mathrm{H} 1 \mathrm{~B}$ & 0.9800 & $\mathrm{C} 1^{\prime}-\mathrm{C} 2{ }^{\prime}$ & $1.5222(15)$ \\
\hline $\mathrm{C} 1-\mathrm{H} 1 \mathrm{C}$ & 0.9800 & $\mathrm{C} 1{ }^{\prime}-\mathrm{H} 1{ }^{\prime} 1$ & 0.9800 \\
\hline $\mathrm{C} 2-\mathrm{N} 5$ & $1.5057(13)$ & $\mathrm{C} 1{ }^{\prime}-\mathrm{H} 1{ }^{\prime 2}$ & 0.9800 \\
\hline $\mathrm{C} 2-\mathrm{C} 6$ & $1.5245(14)$ & $\mathrm{C} 1{ }^{\prime}-\mathrm{H} 1{ }^{\prime} 3$ & 0.9800 \\
\hline $\mathrm{C} 2-\mathrm{C} 3$ & $1.5259(14)$ & $\mathrm{C} 2^{\prime}-\mathrm{N} 5^{\prime}$ & $1.5063(13)$ \\
\hline $\mathrm{C} 3-\mathrm{O} 4$ & $1.4199(13)$ & $\mathrm{C} 2^{\prime}-\mathrm{C}^{\prime}$ & $1.5212(15)$ \\
\hline $\mathrm{C} 3-\mathrm{H} 3 \mathrm{~A}$ & 0.9900 & $\mathrm{C} 2^{\prime}-\mathrm{C} 3^{\prime}$ & $1.5285(16)$ \\
\hline $\mathrm{C} 3-\mathrm{H} 3 \mathrm{~B}$ & 0.9900 & C3'-O4' & $1.4129(15)$ \\
\hline $\mathrm{O} 4-\mathrm{H} 4$ & 0.8400 & C3'-H3'1 & 0.9900 \\
\hline N5-H5A & 0.9100 & $\mathrm{C}^{\prime}-\mathrm{H} 3{ }^{\prime} 2$ & 0.9900 \\
\hline N5-H5B & 0.9100 & O4'--H4' & 0.8400 \\
\hline N5-H5C & 0.9100 & N5'-H5'1 & 0.9100 \\
\hline C6-H6A & 0.9800 & N5'-H5'2 & 0.9100 \\
\hline C6-H6B & 0.9800 & N5'-H5'3 & 0.9100 \\
\hline C6-H6C & 0.9800 & C6'-H6'1 & 0.9800 \\
\hline Mo7-O11 & $1.7594(8)$ & $\mathrm{C6}^{\prime}-\mathrm{H} 6{ }^{\prime} 2$ & 0.9800 \\
\hline Mo7-O9 & $1.7645(8)$ & $\mathrm{C}^{\prime}-\mathrm{H} 6^{\prime} 3$ & 0.9800 \\
\hline \multicolumn{4}{|c|}{ Bond angle $\left({ }^{\circ}\right)$} \\
\hline $\mathrm{C} 2-\mathrm{C} 1-\mathrm{H} 1 \mathrm{~A}$ & 109.5 & $\mathrm{O} 11-\mathrm{Mo} 7-\mathrm{O} 10$ & $110.18(4)$ \\
\hline $\mathrm{C} 2-\mathrm{C} 1-\mathrm{H} 1 \mathrm{~B}$ & 109.5 & O9-Mo7-O10 & $108.07(4)$ \\
\hline $\mathrm{H} 1 \mathrm{~A}-\mathrm{C} 1-\mathrm{H} 1 \mathrm{~B}$ & 109.5 & $\mathrm{O} 8-\mathrm{Mo} 7-\mathrm{O} 10$ & $106.85(4)$ \\
\hline $\mathrm{C} 2-\mathrm{C} 1-\mathrm{H} 1 \mathrm{C}$ & 109.5 & $\mathrm{C} 2^{\prime}-\mathrm{C} 1^{\prime}-\mathrm{H} 1^{\prime} 1$ & 109.5 \\
\hline $\mathrm{H} 1 \mathrm{~A}-\mathrm{C} 1-\mathrm{H} 1 \mathrm{C}$ & 109.5 & $\mathrm{C} 2{ }^{\prime}-\mathrm{C} 1^{\prime}-\mathrm{H} 1^{\prime} 2$ & 109.5 \\
\hline $\mathrm{H} 1 \mathrm{~B}-\mathrm{C} 1-\mathrm{H} 1 \mathrm{C}$ & 109.5 & $\mathrm{H} 1{ }^{\prime} 1-\mathrm{C} 1{ }^{\prime}-\mathrm{H} 1{ }^{\prime} 2$ & 109.5 \\
\hline $\mathrm{N} 5-\mathrm{C} 2-\mathrm{C} 6$ & $108.27(8)$ & $\mathrm{C} 2^{\prime}-\mathrm{C} 1^{\prime}-\mathrm{H} 1^{\prime} 3$ & 109.5 \\
\hline
\end{tabular}

Bull. Chem. Soc. Ethiop. 2013, 27(1) 


\section{RESULTS AND DISCUSSION}

\section{FT- IR studies}

The IR spectra of this compound in $\mathrm{KBr}$ matrix confirm the presence $\mathrm{OH}$ group as a broad band at $2600-3400 \mathrm{~cm}^{-1}$ which is assigned to the intramolecular $\mathrm{H}$-binding vibration $\left(\mathrm{O}-\mathrm{H}^{\cdots} \mathrm{N}\right)[13$, 14]. The bands related to N-H stretching vibrations at $3197 \mathrm{~cm}^{-1}$, the aliphatic C-H vibrations are observed at $2584-2875 \mathrm{~cm}^{-1}$. The $\mathrm{Mo}=\mathrm{O}$ bands are exhibited at 1084 and $1053 \mathrm{~cm}^{-1}$ in this compound [15-18] (Figure 3).

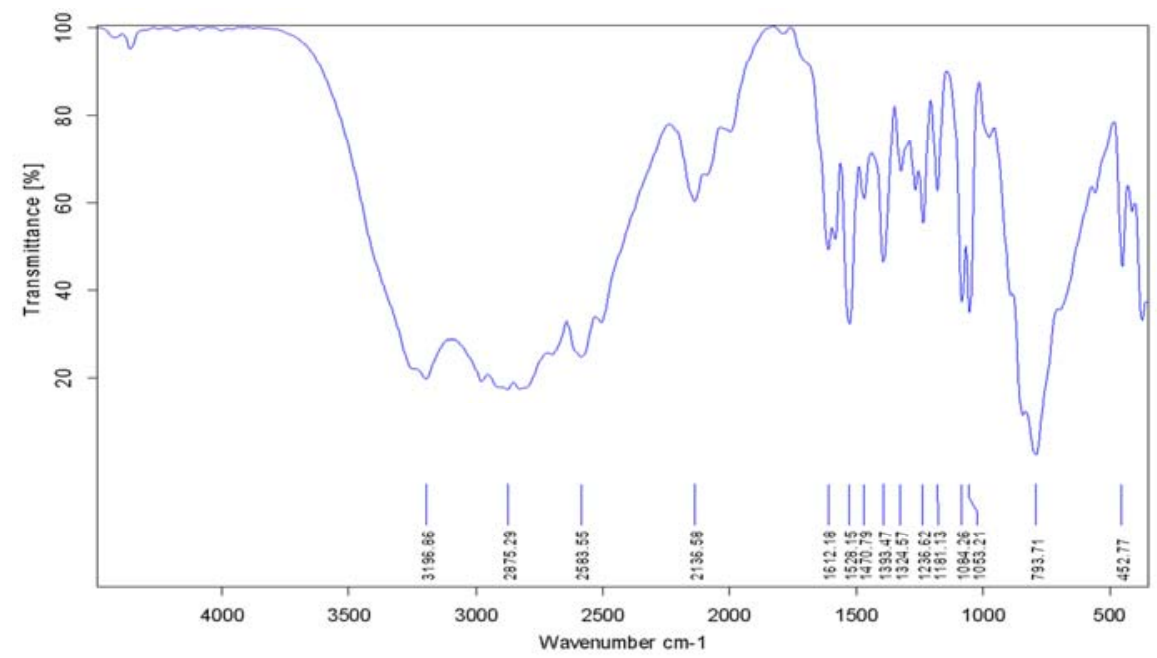

Figure 3. FT-IR spectra of $\left(\mathrm{OHRNH}_{3}\right)_{2}{ }^{2+}\left(\mathrm{MoO}_{4}\right)^{2-}(\mathrm{R}=2$-methyl-1-propyl $)$ as a new molybdate salt.

$U V$ - Vis studies

In order to studying more about the proton transfer compound, the electronic spectra of the components was recorded in methanol as solvent. Mo(VI) and 2- amino-2-methyl-1-propanol show maximum absorbance at 212 and $224 \mathrm{~nm}$, respectively, while the resulting proton transfer compound shows three maxima at 214, 280 and $400 \mathrm{~nm}$. The appearance of a new peak and at larger wavelength is due to the proton transfer from the diacid to the amine and the consequent interaction of the resulting oppositely charged species $[19,20]$.

\section{${ }^{I} H$ NMR studies}

The ${ }^{1} \mathrm{H}$ NMR spectrum of the proton transfer compound shows the presence of a sharp singlet at $3.16 \mathrm{ppm}(\mathrm{s}, 1 \mathrm{H})$ which is rationalized to the $\mathrm{O}-\mathrm{H}$ proton. Two signals that are recorded at 1.17 ppm (s, 3H) and $3.60 \mathrm{ppm}(\mathrm{s}, 2 \mathrm{H})$ in PTC refer to and $\mathrm{CH}_{3}$ and $\mathrm{CH}_{2}$ protons, respectively. The signals related to $\mathrm{N}-\mathrm{H}$ protons appear at $3.30 \mathrm{ppm}(\mathrm{s}, 2 \mathrm{H})$ and $3.78 \mathrm{ppm}(\mathrm{s}, 1 \mathrm{H})$ (Figure 4). 


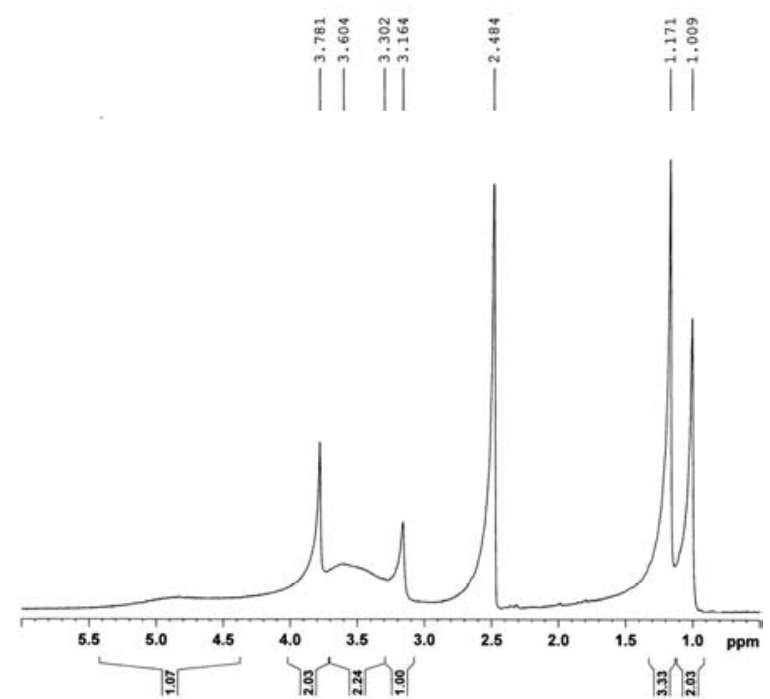

Figure 4. ${ }^{1} \mathrm{H}$ NMR spectra of $\left(\mathrm{OHRNH}_{3}\right)_{2}{ }^{2+}\left(\mathrm{MoO}_{4}\right)^{2-}(\mathrm{R}=2$-methyl-1-propyl $)$ as a new molybdate salt.

\section{Structural analysis}

The molecular structure of the molybdenum salt crystal (Figure 1) consists of discrete $\left(\mathrm{MoO}_{4}\right)^{-2}$ anion and $2(\mathrm{HORNH} 3)^{+1}$ cations. Anion is mononuclear. Table lists selected bond distances and angles. In the anion, the metal cation $\mathrm{Mo}^{\mathrm{VI}}$ is coordinated to four oxygen anions. The Mo cation has a distorted geometry. The bond angles $\mathrm{O}_{11}-\mathrm{Mo}_{7}-\mathrm{O}_{10}\left(110.18^{\circ}\right), \mathrm{O}_{9}-\mathrm{Mo}_{7}-\mathrm{O}_{10}\left(108.07^{\circ}\right)$, $\mathrm{O}_{8}-\mathrm{Mo}_{7}-\mathrm{O}_{10}\left(106.85^{\circ}\right)$, and the bond distances $\mathrm{Mo}_{7}-\mathrm{O}_{8}(1.7695 \AA), \mathrm{Mo}_{7}-\mathrm{O}_{10}\left(1.7731 \AA\right.$ ), $\mathrm{Mo}_{7^{-}}$ $\mathrm{O}_{11}(1.7594 \AA)$ and $\mathrm{Mo}_{7}-\mathrm{O}_{9}(1.7645 \AA)$. Cations are linked by hydrogen bonding to the anions (see Figures 5, 6 and Table 3).

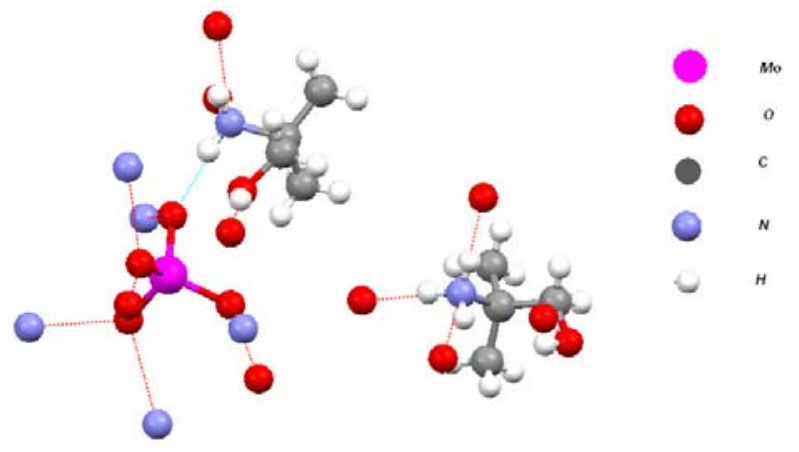

Figure 5. The hydrogen bonding in the structure of $\left.\mathrm{OHRNH}_{3}\right)_{2}{ }^{2+}\left(\mathrm{MoO}_{4}\right)^{2-}(\mathrm{R}=2$-methyl-1propyl) as a new molybdate salt and the cohesion between the different groups is maintained by a three dimensional network of hydrogen bonds. 


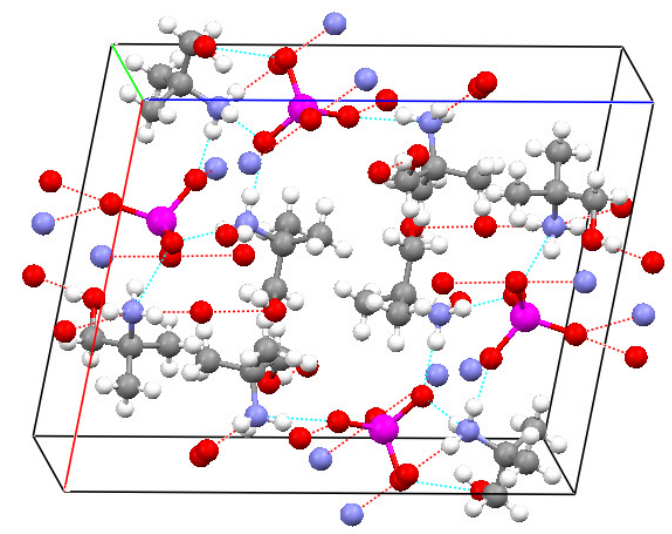

Figure 6. The crystal packing and the hydrogen bonding in the structure of $\left.\mathrm{OHRNH}_{3}\right)_{2}{ }^{2+}\left(\mathrm{MoO}_{4}\right)^{2-}(\mathrm{R}=2$-methyl-1-propyl $)$ crystalline molybdate salt.

Table 3. The hydrogen-bond geometry $\left(\AA,^{\circ}\right)$ of $\left(\mathrm{OHRNH}_{3}\right)_{2}{ }^{2+}\left(\mathrm{MoO}_{4}\right)^{2-}(\mathrm{R}=2$-methyl-1-propyl $)$ crystalline as a new molybdate salt.

\begin{tabular}{|l|l|l|l|l|}
\hline$D-\mathrm{H}^{\prime} \cdots A$ & $D-\mathrm{H}$ & $\mathrm{H} \cdots A$ & $D \cdots A$ & $D-\mathrm{H} \cdots A$ \\
\hline $\mathrm{O} 4-\mathrm{H} 4 \cdots \mathrm{O} 11^{\text {i }}$ & 0.84 & 1.90 & $2.7286(11)$ & 168 \\
\hline $\mathrm{N} 5-\mathrm{H} 5 A \cdots \mathrm{O} 8^{\text {ii }}$ & 0.91 & 1.89 & $2.7917(12)$ & 168 \\
\hline $\mathrm{N} 5-\mathrm{H} 5 B \cdots \mathrm{O} 11^{\text {iii }}$ & 0.91 & 1.99 & $2.8623(12)$ & 160 \\
\hline $\mathrm{N} 5-\mathrm{H} 5 C \cdots \mathrm{O} 9$ & 0.91 & 2.06 & $2.8780(12)$ & 149 \\
\hline $\mathrm{O} 4{ }^{\prime}-\mathrm{H} 4^{\prime} \cdots \mathrm{O} 8^{\text {iv }}$ & 0.84 & 2.07 & $2.6978(12)$ & 131 \\
\hline $\mathrm{N} 55^{\prime}-\mathrm{H}^{\prime} 1 \cdots \mathrm{O} 10^{\mathrm{v}}$ & 0.91 & 1.85 & $2.7553(12)$ & 172 \\
\hline $\mathrm{N} 5^{\prime}-\mathrm{H}^{\prime} 2 \cdots \mathrm{O} 9^{\text {vi }}$ & 0.91 & 1.91 & $2.8181(12)$ & 172 \\
\hline $\mathrm{N} 55^{\prime}-\mathrm{H}^{\prime} 3 \cdots \mathrm{O} 10^{\text {iv }}$ & 0.91 & 1.92 & $2.7867(12)$ & 158 \\
\hline
\end{tabular}

Symmetry codes: (i) $-x+1,-y+2,-z$; (ii) $x, y-1, z$; (iii) $-x+1,-y+1,-z$; (iv) $x+1 / 2,-y+3 / 2, z+1 / 2$; (v) $-x+1,-y+1,-$ $z+1$; (vi) $x+1 / 2,-y+1 / 2, z+1 / 2$.

\section{CONCLUSION}

In summary, this article presents the synthesis and X-ray structure of a new molybdate salt as a single crystal. We have demonstrated that the proton transfer compound can be used as an appropriate starting material to synthesize a variety of interesting molybdenum compound or a molybdate salt, showing different complexation behavior of this proton transfer system as discussed previously.

\section{Supplementary data}

Crystallography data (excluding structure factors) for the structure reported in this paper has been deposited with the Cambridge Crystallographic Center, CCDC No. 893992. Copies of the data can be obtained free of charge on application to The Director, CCDC, 12 Union Road, Cambridge CB2 1EZ, UK, fax: +44 1223336 033, e-mail: deposit@ccdc.cam.ac.uk or http://www.ccdc.cam.ac.uk

\section{ACKNOWLEDGEMENTS}

Shahid Bahonar University of Kerman is acknowledged for support of this work.

Bull. Chem. Soc. Ethiop. 2013, 27(1) 


\section{REFERENCES}

1. Glaser, R.; Lewis, M. Org. Lett. 1999, 1, 273.

2. Chipanina, N.N.; Aksamentova, T.N.; Adamovich, S.N.; Albanov, A.I.; Mirskova, A.N; Mirskov, R.G.; Voronkov, M.G. Comput. Theor. Chem. 2012, 985, 36.

3. He, F.; Ramirez, J.; Lebrilla, C.B. J. Am. Chem. Soc. 1999, 121, 4726.

4. (a) Tarkka, R.M.; Zhang, X.; Jenekhe, S.A. J. Am. Chem. Soc., 1996, 118, 9438. (b) Videnova-Adrabińska, V. J. Mol. Struc.: THEOCHEM 1996, 374, 199.

5. Nogami, M.; Daiko, Y.; Akai, T.; Kasuga, T. J. Phys. Chem. B 2001, 105, 4653.

6. Kresge, A.J.; Powell, M.F. J. Am. Chem. Soc. 1981, 103, 972.

7. Ramezanipour, F.; Aghabozorg, H.; Shokrollahi, A.; Shamsipur, M.; Stoeckli-Evans, H.; Soleimannejad, J.; Sheshmani, S.h. J. Mol. Struc. 2005, 779, 77.

8. Swift, J.; Pivovar, A.M.; Reynolds, A.M.; Ward, M.D. J. Am. Chem. Soc. 1998, 120, 5887.

9. MacDonnald, J.C.; Dorrestein, P.C.; Pilley, M.M.; Foote, M.M; Lundburg, J.L; Henning, R.W.; Schultz, A.J.; Manson, J.L. J. Am. Chem. Soc. 2000, 122, 11692.

10. (a) Moghimi, A.; Shokrollahi, A.; Shamsipur, M.; Aghabozorg, H.; Ranjbar, M. J. Mol. Struc. 2004, 701, 49. (b) Li-Kai Yan, Shi-Zheng Wen, Jian-Ping Wang, Zhong-Min Su. Comput. Theor. Chem. 2012, 988, 1.

11. ${ }^{\mathrm{a}}$ Bruker SAINT Bruker AXS Inc.: Madison, Wisconsin, USA; 2008. ${ }^{\mathrm{b}}$ Bruker APEX2 Bruker AXS Inc.: Madison, Wisconsin, USA; 2009. Sheldrick, G.M. Acta Cryst. 2008, A64, 112. ${ }^{\mathrm{d}}$ Sheldrick, G.M. SADABS University of Göttingen: Germany; 2008.

12. Sheldrick, G.M. SHELXS97 and SHELXL97, University of Göttingen: Germany; 1997.

13. Kumar, D.N.; Garg, B.S. Spectrochim. Acta Part A 2006, 64, 141.

14. Dutt, N.K.; Nag, K. J. Inorg. Nucl. Chem. 1968, 30, 2493.

15. Chai, F.L.; Su, H.L.; Wang, X.Y.; Tao, J.C. Inorg. Chim. Acta 2009, 362, 3840.

16. Bagherzadeh, M.; Latifi, R.; Tahsini, L.; Keith Woo, L. Inorg. Chim. Acta 2009, 362, 3698.

17. Rezaeifard, A.; Sheikhshoaie, I.; Monadi, N.; Alipour, M. Polyhedron 2010, 29, 2703.

18. Sheikhshoaie, I.; Rezaeifard, A.; Monadi, N.; Kaafi, S. Polyhedron 2009, $28,733$.

19. Sadeghi, S.; Alizadeh, N.; Shamsipur, M. J. Inclusion Phenom. 1999, 34, 431; and references therein.

20. Moghimi, A.; Moosavi, S.M.; Kordestani, D.; Maddah, B.; Shamsipur, M.; Aghabozorg, H.; Ramezanipour, F.; Kickelbick, G. J. Mol. Struc. 2007, 828, 38. 